\title{
COVID-19 complicating perioperative management of LVAD implantation: a case report and systematic review
}

\author{
Deborah Belfort ${ }^{1}$, Bruno Biselli ${ }^{1}$, Monica Ávila ${ }^{1}$, Maria Tereza Lira ${ }^{1}$, Filomena Galas ${ }^{1}$, \\ Samuel Steffen ${ }^{1}$, Fabio Gaiotto ${ }^{1}$, Fábio Jatene ${ }^{1}$, Edimar Bocchi ${ }^{1}$, and Silvia Ferreira ${ }^{1}$ \\ ${ }^{1}$ Universidade de São Paulo Instituto do Coração
}

March 30, 2021

\begin{abstract}
The coronavirus 2019 disease (COVID-19) affected 125 million people worldwide and caused 2.7 million deaths. Some comorbidities are associated with worse prognosis and left ventricular assist device (LVAD) recipients are probably part of this high-risk population. We report a 31-year-old male patient who developed COVID-19 during LVAD implantation. His postoperative period was complicated by severe pneumonia and mechanical ventilation leading to right ventricular failure (RVF) and inotrope necessity. He experienced multiple complications, but eventually recovered. We present a systematic review of LVAD recipients and COVID-19. Among 14 patients, the mean age was 62.7 years, $78.5 \%$ were male. Five patients $(35.7 \%)$ required mechanical ventilation and 3 patients $(21.4 \%)$ died. Two patients $(14.2 \%)$ had thromboembolic events. This case and systematic review suggest LVAD recipients are at particular risk of unfavorable outcomes and they may be more susceptible to RVF in the setting of COVID-19, particularly during perioperative period.
\end{abstract}

\section{Introduction}

The coronavirus 2019 disease (COVID-19) affected 125 million people worldwide and caused 2.7 million deaths so far ${ }^{1}$. Some conditions such as diabetes, hypertension, heart failure and coronary artery disease are associated with worse prognosis ${ }^{2}$. Left ventricular assist device (LVAD) recipients are probably part of this high-risk population since they usually have multiple comorbidities and live in a functionally immunocompromised state ${ }^{3}$.

There are few data on LVAD recipients and COVID-19, and most cases occurred months or years after LVAD implantation $^{4-11}$. We present a case of a patient who presented fever during LVAD implantation as the first manifestation of COVID-19 who developed critical disease.

\section{Case report}

A 31-year-old male patient without comorbidities was admitted due to cardiogenic shock and pneumonia. He required orotracheal intubation, dobutamine and norepinephrine on the $2^{\text {nd }}$ hospitalization day. On the $8^{\text {th }}$ hospitalization day, worsening of hemodynamics led to necessity of intra-aortic balloon pump (IABP) and extracorporeal membrane oxygenation (ECMO).

Respiratory symptoms started 9 days before hospitalization. Nasopharyngeal SARS-CoV-2 reverse transcription polymerase chain reaction (RT-PCR) were performed on admission and $2^{\text {nd }}$ day of hospitalization. Two SARS-CoV-2 RT-PCR in tracheal secretion were also performed on $3^{\text {rd }}$ and $4^{\text {th }}$ days, both negative. An IgM and IgG SARS-CoV-2 test was negative after 23 days of symptoms. Thoracic computed tomography scan (CT scan) suggested bacterial pneumonia (Figure 1). Echocardiogram showed severe left ventricular dysfunction (ejection fraction 24\%) and moderate right ventricular dysfunction (RVD). Endomyocardial biopsy 
revealed diffuse hypertrophy and mild fibrosis. Cardiac magnetic resonance revealed no late gadolinium enhancement.

After initial support and antibiotics, the patient recovered from organ dysfunctions. He was extubated and ECMO was weaned off after 9 days. However, he persisted dependent on dobutamine and IABP, and LVAD implantation was planned as a bridge to transplant. Right heart catheterization revealed no pulmonary hypertension (Table 1).

A Heart Mate 3 was successfully implanted on-pump on $83^{\text {rd }}$ day of hospitalization. However, the patient presented fever in the operating room and next 3 days. He was extubated on $2^{\text {nd }}$ postoperative day (POD) and was weaned off vasopressors. He also presented lymphopenia and C-reactive protein elevation (Table 1). Nasopharyngeal SARS-CoV-2 RT-PCR returned positive.

On $5^{\text {th }}$ POD, the patient became hypoxemic requiring high flow nasal cannula (chest X-ray on Figure 2). He was on intravenous heparin since $2^{\text {nd }} \mathrm{POD}$ and antibiotics and corticosteroids were prescribed. Blood and tracheal secretion urine cultures returned negative. Echocardiogram revealed severe RVD. Hypotension and a drop on central venous saturation (Table 2) led to milrinone $0.75 \mathrm{mcg} / \mathrm{kg} / \mathrm{min}$ and dobutamine $20 \mathrm{mcg} / \mathrm{kg} / \mathrm{min}$ requirement.

On $10^{\text {th }}$ POD endotracheal intubation was required. The patient experienced severe acute respiratory distress syndrome (ARDS) and multiple complications: shock requiring vasopressors, secondary bacterial pneumonia, hemothorax, catheter-related bloodstream infection and pneumothorax due to barotrauma. A CT scan performed on $34^{\text {th }}$ POD revealed bilateral ground glass opacities, bilateral pneumothorax and consolidation (Figure 1.B). He had no thromboembolic events.

After 48 days of LVAD implantation, he was free from mechanical ventilation (MV) and inotropes. He was discharged from the intensive care unit after 51 days and from the hospital after 199 days of hospitalization.

\section{Discussion}

We present a case of a previously healthy young man who presented with cardiogenic shock of unknown etiology and respiratory failure with multiple negative SARS-CoV-2 RT-PCRs. He required LVAD implantation, and postoperative period was complicated by nosocomial SARS-CoV-2 pneumonia. To the best of our knowledge this is the first case report of COVID-19 in the immediate postoperative period of LVAD implantation. In this report we demonstrate that COVID-19 was associated with RVF in the setting of respiratory failure.

Right ventricular failure complicates 4-50\% of LVAD implants ${ }^{12}$, and our patient was just in this delicate phase when he developed COVID-19. Although RVF can be a consequence of LVAD implantation, the patient did not present clinical or echocardiographic signs of RVF until he required noninvasive MV. Therefore, COVID-19 probably had a main contribution to RVF in this case.

The new coronavirus is associated with RVF by multiple mechanisms. Severe pneumonia and ARDS may lead to $\mathrm{RVF}^{14}$. The pathophysiology is multifactorial: hypoxia, vascular alterations, MV and hypercapnia all together may lead to pulmonary hypertension and RVF ${ }^{14}$. Myocardial injury has also been described in COVID-19, worsening biventricular function ${ }^{13}$. Our patient presented extreme troponin elevation after LVAD implantation, which could be due to the surgical procedure or a superimposed myocardial injury secondary to the new coronavirus.

A systematic review was performed searching PubMed and MEDLINE and using the keywords "LVAD" and "COVID-19" for comparison with this case. The selection criteria were case reports or case series of LVAD recipients and positive SARS-CoV-2 RT-PCR. Pre-specified demographic and prognostic data was collected. A total of 157 publications were found, and 8 publications met the selection criteria.

Fourteen patients are described (Table 3). The mean age was 62.7 years and $78.5 \%$ were male. The time since LVAD implant varied from 0.03 months to 6.8 years, and none of them happened in the immediate 
postoperative period. Five patients (35.7\%) required MV and 3 patients (21.4\%) died. Two patients (14.2\%) had thromboembolic events. Six patients $(42.8 \%)$ had mild symptoms, and three were followed as outpatients.

As expected, LVAD patients with COVID-19 had higher mortality than general population. Data from China shows a mortality rate of $2.3 \%^{2}$, while in this systematic review LVAD recipients had a fatality rate of $21.4 \%$, probably because they have multiple comorbidities and live in a functionally immunocompromised state $^{3}$. In contrast, our patient eventually recovered despite multiple complications, probably because of young age and lack of other comorbidities.

Mechanical ventilation was required in $35.7 \%$ of patients, while in general population only $14 \%$ present pneumonia and $5 \%$ require $\mathrm{MV}^{2}$. Despite frequent need for MV in LVAD patients and the concern about RVF in this population, in this systematic review only one patient presented RVF requiring inotropes ${ }^{6}$. This patient had mild symptoms of COVID-19 and did not present SARS-CoV-2 pneumonia or myocardial injury, so that RVD was not thought to be related to COVID-19.

The new coronavirus is also associated with high thromboembolic rates, and studies reported thromboembolism incidence of $25 \%$ in critical patients ${ }^{15}$. LVAD recipients in parallel are in intrinsic risk of pump thrombosis. This may raise a concern about thromboembolic events (TE) in this population. However, chronic anticoagulation may play a protective role since so far only $14.2 \%$ had TE while infected ${ }^{5,8}$.

This study has limitations. All publications are case reports or case series. There is clinical heterogeneity between patients regarding age, type of LVAD and time since LVAD implantation. Mild cases of COVID19 might be underrepresented. Despite these limitations, this is the first systematic review of LVAD and COVID-19. Also, the present case report illustrates the vulnerability of LVAD recipients regarding RVF in the perioperative setting.

In this systematic review, LVAD recipients with COVID-19 had a higher mortality and morbidity than general population. More study is needed to better understand COVID-19 in LVAD recipients.

\section{Legends}

Figure 1. A. Thoracic Computed Tomography (CT) scan on admission revealing consolidation on right lung. B. Thoracic CT scan on postoperative day 34 revealing multiple ground glass opacities, diffuse consolidations and moderate bilateral pneumothorax.

Figure 2. Chest radiography on postoperative day 5 revealing multiple bilateral opacities.

\section{References}

1. Organization WH. WHO Coronavirus Disease (COVID-19) Dashboard. Accessed 27/03/2021, https://covid19.who.int/

2. Wu Z, McGoogan JM. Characteristics of and Important Lessons From the Coronavirus Disease 2019 (COVID-19) Outbreak in China: Summary of a Report of 72314 Cases From the Chinese Center for Disease Control and Prevention. JAMA . Feb 2020;doi:10.1001/jama.2020.2648

3. Ankersmit HJ, Edwards NM, Schuster M, et al. Quantitative changes in T-cell populations after left ventricular assist device implantation: relationship to T-cell apoptosis and soluble CD95. Circulation. Nov 9 1999;100(19 Suppl):Ii211-5. doi:10.1161/01.cir.100.suppl_2.ii-211

4. Chau VQ, Oliveros E, Mahmood K, et al. The Imperfect Cytokine Storm: Severe COVID-19 With ARDS in a Patient on Durable LVAD Support.JACC: Case Reports . 2020/07/15/ 2020;2(9):1315-1320. doi:https://doi.org/10.1016/j.jaccas.2020.04.001

5. Sobol I, Yuzefpolskaya M, Roth Z, et al. Characteristics and Outcomes of Patients With a Left Ventricular Assist Device With Coronavirus Disease-19. J Card Fail . 10 2020;26(10):895-897. doi:10.1016/j.cardfail.2020.09.011 
6. Piperata A, Bottio T, Gerosa G. COVID-19 infection in left ventricular assist device patients. J Card Surg. Aug 2020;doi:10.1111/jocs.14969

7. Loforte A, Gliozzi G, Martin Suarez S, Pacini D. Contributory Role of Positron Emission Tomography in a Left Ventricular Assist Device Recipient at the Time of COVID-19 Pandemic. ASAIO J . 06 2020;66(6):599602. doi:10.1097/MAT.0000000000001176

8. Hodges K, Mubashir M, Insler J, et al. Successful management of COVID-19 and associated coagulopathy in a patient with durable left ventricular assist device. J Card Surg . Aug 2020;doi:10.1111/jocs.14937

9. Korada SKC, Mann JA, Hasan AK, et al. Management of COVID-19 in a durable left ventricular assist device recipient: A continuity of care perspective. Heart Lung . Aug 2020;49(6):688-691. doi:10.1016/j.hrtlng.2020.08.012

10. Singh R, Domenico C, Rao SD, et al. Novel Coronavirus Disease 2019 in a Patient on Durable Left Ventricular Assist Device Support. J Card Fail . 05 2020;26(5):438-439. doi:10.1016/j.cardfail.2020.04.007

11. Mahmood K, Rashed ER, Oliveros E, et al. Predisposition or Protection?: COVID-19 in a Patient on LVAD Support With HIV/AIDS.JACC Case Rep . C) 2020 The Authors.; 2020:1337-1341. vol. 9.

12. Soliman OII, Akin S, Muslem R, et al. Derivation and Validation of a Novel Right-Sided Heart Failure Model After Implantation of Continuous Flow Left Ventricular Assist Devices: The EUROMACS (European Registry for Patients with Mechanical Circulatory Support) Right-Sided Heart Failure Risk Score. Circulation . 02 2018;137(9):891-906. doi:10.1161/CIRCULATIONAHA.117.030543

13. Shi S, Qin M, Shen B, et al. Association of Cardiac Injury With Mortality in Hospitalized Patients With COVID-19 in Wuhan, China.JAMA Cardiol . 07 2020;5(7):802-810. doi:10.1001/jamacardio.2020.0950

14. Bunge JJH, Caliskan K, Gommers D, Reis Miranda D. Right ventricular dysfunction during acute respiratory distress syndrome and veno-venous extracorporeal membrane oxygenation. J Thorac Dis . Mar 2018;10(Suppl 5):S674-S682. doi:10.21037/jtd.2017.10.75

15. Al-Ani F, Chehade S, Lazo-Langner A. Thrombosis risk associated with COVID-19 infection. A scoping review. Thromb Res . 08 2020;192:152-160. doi:10.1016/j.thromres.2020.05.039

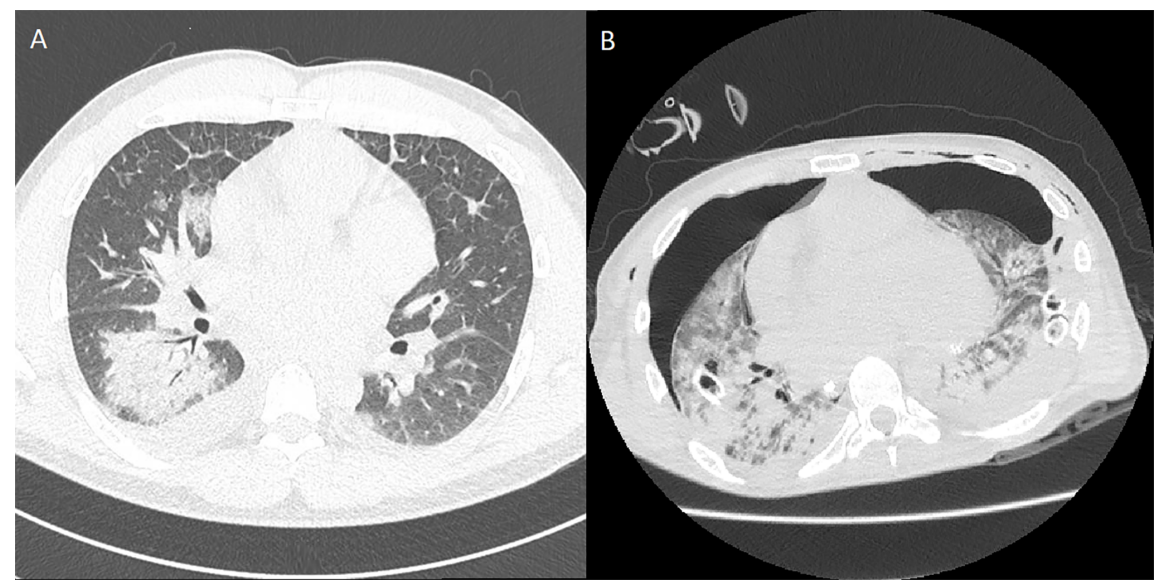




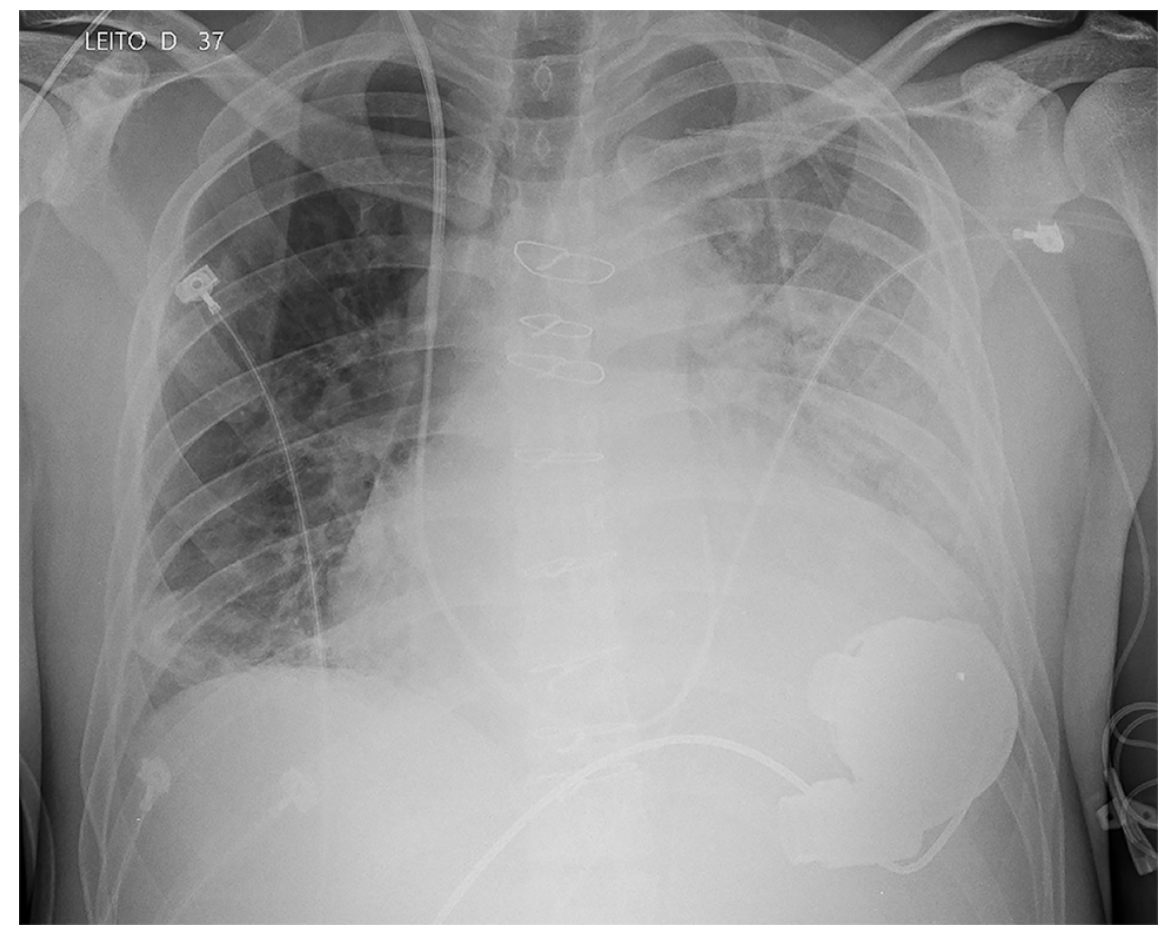

\section{Hosted file}

Table_1.pdf available at https://authorea.com/users/404906/articles/515993-covid-19complicating-perioperative-management-of-lvad-implantation-a-case-report-and-systematicreview

\section{Hosted file}

Table_2.pdf available at https://authorea.com/users/404906/articles/515993-covid-19complicating-perioperative-management-of-lvad-implantation-a-case-report-and-systematicreview

\section{Hosted file}

Table_3.pdf available at https://authorea.com/users/404906/articles/515993-covid-19complicating-perioperative-management-of-lvad-implantation-a-case-report-and-systematicreview 